\title{
Programas de transferencia condicionada y representaciones de la pobreza en Bolivia y Perú
}

\author{
Nora NAGELS \\ Université du Québec à Montréal (UQAM)-Canadá \\ nagels.nora@uqam.ca
}

Recibido: 27-05-2014

Aceptado: 01-10-2015

\section{Resumen}

Bolivia y Perú adoptaron los mismos instrumentos de política social — los programas de transferencias condicionadas - para responder a problemas públicos similares bajo regímenes políticos diferentes. A partir de una metodología cualitativa de análisis de discurso, este artículo investiga las representaciones de la pobreza y del Estado construidas por los actores claves de estos programas sociales. Subrayando las diferencias más que las similitudes, se demuestra que los mismos instrumentos de política social se vinculan a representaciones sociales opuestas de la pobreza y del rol del Estado en cada país. La principal explicación de esto es que, lejos de ser impuestos desde el exterior, estos programas son apropiados y adaptados por cada régimen político.

Palabras clave: pobreza; focalización; transferencias condicionadas; análisis del discurso; indigenismo. 


\title{
Conditional Cash Transfer Programs and the Representations of Poverty in Bolivia and Peru
}

\begin{abstract}
Bolivia and Peru adopted the same instruments of social policy - conditional cash transfer programs - to solve the same public problems under different political regimes. By means of the qualitative methodology of discourse analysis, this paper studies the representations of poverty and State made by key actors of those social programs. Underlying more differences than similarities, one demonstrates that the same social policy is linked to opposite social representations of poverty and the State role in every country. The main explanation for this is, far from being imposed by international organizations, those programs are adopted and adapted by each political regime.
\end{abstract}

Keywords: Poverty; Focalization; Conditional Cash Transfer Program; Discourse Analysis; Indigenism.

\section{Referencia normalizada}

NAGELs, N. (2015): "Programas de transferencia condicionada y representaciones de la pobreza en Bolivia y Perú “, Política y Sociedad, 52 (3), pp. 771-791.

Sumario: 1. Introducción. 2. Contexto histórico. 3. Las representaciones sociales de la pobreza.

4. Conclusiones. 5. Bibliografía. 6. Anexos.

Agradezco a Martin Mendoza-Botelho por la corrección de una versión preliminar de este texto. Este artículo fue presentado en el congreso anual de la Asociación internacional de ciencias políticas (IPSA/AISP) en julio 2012 y una parte de sus resultados fue publicada en Nagels (2014a). 


\section{Introducción}

Los programas de transferencias condicionadas (PTCs) tienen dos objetivos: a corto plazo, buscan reducir la pobreza aumentando la liquidez en la economía diaria de las familias beneficiarias y, a largo plazo, buscan romper el ciclo intergeneracional de la pobreza desarrollando el capital humano de las generaciones futuras. Para lograrlo, transfieren subsidios monetarios a las familias pobres condicionándolos a la escolarización de los niños y niñas y al seguimiento sanitario de la maternidad. Desde la implantación del programa Progresa/Oportunidades en México en 1997 y el Bolsa Familia en Brasil en 2003, los PTCs proliferan en el continente Latino-americano y, en general, en los países llamados "en vía de desarrollo".

Los PTCs son vistos como el mayor cambio en las políticas sociales de América Latina en los últimos 20 años (Barrientos, 2009; Martínez, 2008) y se consideran como propuestas formuladas desde la perspectiva de inversión social (Jenson, 2010; Staab, 2010; Razavi, 2014). La perspectiva de la inversión social da forma a la estructura cognitiva de la política social tanto en el Sur como en el Norte, y se fundamenta en promover la inversión en el capital humano de las generaciones futuras (Jenson y Saint-Marti, 2006). Esta perspectiva defiende la vuelta de la intervención del Estado al ámbito social reconociendo que el mercado, y más concretamente el mercado laboral, no permite garantizar el bienestar, aunque el objetivo del Estado no es tanto proteger a los ciudadanos de los riesgos sociales, sino prevenir tales riesgos (Morel et al., 2011).

Buena parte de la literatura actual afirma que los PTCs pertenecen al ámbito de la inversión social y alcanzan sus objetivos mediante la inversión en el capital humano de la niñez. Así, las organizaciones financieras internacionales como el Banco Mundial, el Fondo Monetario Internacional, las agencias de la Organización de las Naciones Unidas — como el PNUD, la OIT y la CEPAL—, los organismos independientes como el IFRI, el Wilson Center e incluso instituciones académicas, estiman que los PTCs son eficaces para alcanzar sus objetivos en educación (OIT et Bachelet, 2011; Barrientos y Dejong, 2006; Fiszbein et al., 2009), salud (Villatoro, 2004; Skoufias et al., 2001), pobreza y desigualdades (The economist, 2010; Cecchini y Madariaga, 2011; Bastagli, 2009). Como destaca Sugiyama (2011: 262), el resultado de ello es que los actores nacionales identifican a los PTCs como la "nueva norma profesional en la comunidad (de los teóricos) del desarrollo económico".

Perú y Bolivia no son una excepción. Aunque con regímenes políticos opuestos, el gobierno peruano de Alan García y el boliviano de Evo Morales, casi al mismo tiempo, adoptaron esta nueva "norma profesional". Como resultado, se alinearon con la perspectiva del enfoque de la inversión social y el Estado ganó un nuevo rol en lo social. Más allá de estas similitudes, el objetivo de este artículo es mostrar que el rol del Estado diverge en cada país. Pues, lejos de ser impuestos desde las organizaciones internacionales y ser adoptados tal cual al nivel nacional, los PTCs son traducidos y adaptados 
por los regímenes políticos, que los cambian en función de sus orientaciones políticas. Por tanto el "efecto régimen político" entra en juego en este análisis. ${ }^{1}$

Para estudiar este efecto político, utilizamos en este trabajo el concepto de "instrumentos de políticas públicas" definido por Lascoumes y Le Galès (2005). Concebidos como instrumentos de las políticas sociales, los PTCs pueden ser considerados como dispositivos que organizan las relaciones entre el Estado y la sociedad. Lascoumes y Le Galès (2005: 14) definen un instrumento de acción política "como un dispositivo social y técnico con vocación genérica que da soporte a concepciones concretas de las relaciones política/sociedad y tiene una naturaleza regulatoria”. Para precisar más las concepciones vinculadas a los PTCs, utilizamos en este trabajo la noción de representaciones sociales de Jodelet (1989). Este concepto pone de relieve el proceso de construcción de la realidad social por los actores desde sus posiciones sociales. Según Jodelet (1989: 32), "las representaciones sociales se producen en los discursos. Se realizan por las palabras transmitidas en los mensajes e imágenes mediáticos y cristalizan en las conductas y en los arreglos materiales o espaciales". Los discursos en los que aparecen las representaciones sociales tienen diversos soportes: el lenguaje oral, los documentos, los textos legales, etc. En esta investigación, se recurre al lenguaje oral mediante entrevistas con el fin de poner de manifiesto las relaciones de poder en el proceso de construcción de las representaciones sociales de la pobreza. De hecho, como dice Bourdieu, el lenguaje oral y, por tanto, las palabras son cruciales en la estructura de las relaciones de poder. Según él "se debe examinar el papel desempeñado por las palabras en la construcción de las cosas sociales, así como la contribución que la lucha de la clasificación, dimensión de cualquier lucha de clases, lleva a la formación de las clases: clases de edad, clases de sexo o clases sociales" (Bourdieu, 1982: 99). Por lo tanto, el lenguaje es considerado como un acto de poder y también como algo que participa en la construcción de la realidad. De acuerdo con Bourdieu (1982: 103) "decir es hacer". En consecuencia, al analizar las representaciones sociales podemos acercarnos a la realidad ya que el discurso es considerado como performativo.

En este trabajo se accede a las representaciones sociales de los PTCs a partir del análisis de discurso de sus actores claves. Se realizaron cincuenta entrevistas con protagonistas institucionales involucrados en los PCTs peruanos y bolivianos que participaron tanto en la concepción de los mismos como en su implementación. Se trata de funcionarios públicos (funcionarios o representantes electos) en el gobierno central, regional o local; de expertos (académicos o de centros de estudios vinculados a los ministerios) y de profesionales a cargo de la gestión de los programas (promotores, maestros y médicos). Estas entrevistas fueron realizadas en las ciudades de La Paz y

${ }^{1}$ Siguiendo el enfoque de género he mostrado las similitudes entre los PTC peruanos y bolivianos: pertenecen a regímenes de bienestar de inversión social familiarista (Nagels a futuro), son maternalistas (Nagels 2014b) y neocoloniales (Nagels 2014c). 
El Alto en Bolivia, y de Lima y Ayacucho en Perú durante viajes de 3 a 6 meses entre septiembre 2008 y agosto $2010^{2}$. Si bien en este artículo solo se consideran las entrevistas con actores institucionales, otras realizadas con receptores de los PTC fueron analizadas en mi investigación doctoral ${ }^{3}$.

En concreto, los discursos se analizaron mediante el método de análisis de contenido. Su especificidad consiste en asumir que el discurso no es sólo un alineamiento de frases sucesivas sino que, por el contrario, cada acto discursivo refleja unas representaciones sociales que hay que descubrir (Quivy et Van Campenhoudt, 1995). Las entrevistas fueron analizadas con el apoyo del software de análisis cualitativo Atlas.ti, con el que diferentes códigos - como "pobreza" y "Estado"- fueron considerados en la totalidad de las entrevistas transcritas. Aunque el empleo de este software no substituye a la interpretación de los datos, sin embargo ha permitido explicitar las facetas poco visibles, aunque muy intuitivas, del proceso del análisis: describir, organizar y concentrar los datos (Henning y Van Rensburg, 2004).

Este artículo se divide en dos partes. Primero los contextos y los PTCs son presentados brevemente. Después son analizadas las diferencias entre las representaciones de la pobreza, de los pobres y del Estado. En la medida en que el enfoque comparativo resalta las similitudes y las diferencias, conduce a la formulación de hipótesis explicativas (Hassenteufel, 2000). Como resultado, las diferencias entre las representaciones de la pobreza, que en los dos países están vinculadas al mismo instrumento de política social, demuestra el "efecto régimen político". Los PTCs son promovidos pero no impuestos por los organismos internacionales; empero, son apropiados por los gobiernos nacionales según sus propias representaciones del problema público "pobreza". El contexto socio-político explica por qué en Bolivia estas representaciones son de corte nacional-popular y en Perú son más individualistas.

\section{Contexto histórico}

\subsection{Los Bonos en Bolivia}

Teniendo en cuenta las principales reivindicaciones de los movimientos sociales que los llevaron al poder, las primeras reformas del Movimiento al Socialismo (MAS) y del gobierno de Evo Morales (desde 2006) consistieron en la "nacionalización" de los hi-

${ }^{2}$ El periodo cubierto por la investigación se restringe a los dos primeros gobiernos de Evo Morales (2006-2014) en Bolivia y a los de Alejandro Toledo (2001-2006) y Alan García (2006-2011) en Perú.

${ }^{3}$ Titulada "Género y políticas de lucha contra la pobreza en Perú y Bolivia: los desafíos de ciudadanía", mi investigación doctoral analiza sistemáticamente 118 entrevistas con los actores de los PTC, desde los responsables políticos a las receptores (Nagels, 2013). 
drocarburos y en la aprobación de un nueva constitución. En pocas palabras, la primera medida permitió que el Estado pudiera romper su dependencia de la ayuda internacional y financiar las políticas públicas, entre otros, los Bonos; aunque en realidad la "nacionalización" consistió en una renegociación de los contratos con las transnacionales explotadoras de los hidrocarburos más que en una expropiación (Gray Molina, 2007). Por otro lado la nueva Constitución del Estado Plurinacional de Bolivia ha reconocido un nuevo sujeto político: el pueblo indígena, originario y campesinos ${ }^{4}$. Además, de esta Constitución ha surgido el Plan Nacional de Desarrollo "Bolivia Digna, Soberana, Productiva y Democrática para Vivir Bien" (PND) que configura "una política de protección social de desarrollo integral comunitario" con el objetivo de erradicar la extrema pobreza. Aunque esta nueva política incluye proyectos a largo plazo, tales como "Comunidad en Acción", "Plan Vida" o el "Plan de Erradicación de la Extrema Pobreza" (PEEP), sólo los Bonos y la Renta Dignidad han sido concretados. Ello ha sido debido en buena parte a los debates teóricos y las pugnas entre diferentes modelos de desarrollo dentro del Ministerio de Planificación del Desarrollo (Canavire-Bacarreza, 2010: 36).

El Bono Juancito Pinto (BJP) fue creado en $2006^{6}$. Se enfoca hacia todos los niños y niñas de los primeros ocho niveles de la educación pública primaria, aunque se contrapone a la recomendación del Banco Mundial que proponía focalizarlo sobre los más pobres 7 . El BJP tiene por objetivo luchar contra el absentismo escolar y el trabajo infantil. Concede alrededor de 30 \$US al año a los niños, condicionando la entrega del dinero a su inscripción en la enseñanza pública y a que terminen el año escolar con un nivel de asistencia de al menos el 80\% de los días (UE 2008: 1). Este Bono cubre a un $17,5 \%$ de la población total; de la cual el $32,4 \%$ son pobres y el $59,7 \%$ son indigentes (Cecchini et Madariaga, 2011). El presupuesto anual para el año 2010 fue de 54,5 millones \$US (Weisbrot et al., 2009: 16), lo que representa un 1,16\% del PIB (Marco Navarro 2013: 27).

El Bono Juana Azurduy (BJA), identificado como un Bono madre-niño, constituye el mínimo común denominador de los proyectos de política social no concretados por

${ }^{4}$ El artículo 30 de la Constitución Política del Estado Plurinacional crea una nueva categoría de "nación y pueblo indígena, originario y campesinos" que constituyen "toda la colectividad humana que comparte identidad cultural, idioma, tradición histórica, instituciones, territorialidad y cosmovisión, cuya existencia es anterior a la invasión colonial española". Como lo destaca Lacroix (2011: 7), este articulo unifica "categorías socio-étnicas regionales históricas para superar las diferenciaciones terminológicas (originario o indígenas o campesina) y políticos (pueblos o naciones)". En este trabajo el término "indígena" se refiere a esta categoría.

${ }^{5}$ Creado por el Decreto Supremo no 29272 del 12 de septiembre de 2007.

${ }^{6}$ Decreto Supremo nº 28899 del 26 de octubre 2006.

${ }^{7}$ Entrevista el 30 de agosto 2010 con la primera responsable del BJP. 
el gobierno. El BJA fue creado en mayo $2009^{8}$. Su origen está en el proyecto piloto de un "bono madre-niño", que los gestores del PEEP elaboraron con el apoyo técnico del Banco Mundial, focalizado en los 52 municipios más pobres del país que el Presidente decidió extender a todo el país 9 . Madres o mujeres gestantes reciben el equivalente de 260 \$US en un periodo de 33 meses, los cuales son transferidos durante los controles prenatales, el parto institucional y los controles postnatales. El BJA constituye un 1,41\% de los gastos sociales, que representan un $0,22 \%$ del PIB. El BJA se dirige a $3.5 \%$ de la población, que es el $6 \%$ de los pobres y el $10 \%$ de los pobres extremos (Cecchini et Madariaga, 2011). También emplea a alrededor de 600 médicos en exclusiva, a fin de garantizar su aplicación y seguimiento.

Esta investigación analiza los discursos de los actores que han participado en la gestión de los bonos y también los de los que elaboraron los distintos proyectos no concretados (tal como el PEEP), ya que acabaron determinando la configuración del BJA.

\subsection{Juntos en Perú}

El programa Juntos fue creado en $2005^{10}$ durante la presidencia de Alejandro Toledo (2001-2006). Dos hechos principales explican la adopción de este programa. Primero, desde el retorno a la democracia en 2000 — después de la guerra interna y el régimen autoritario de Fujimori entre 1990 y 2000 - el Perú experimentó un proceso de crecimiento económico pero sin redistribución ${ }^{11}$; situación que llevó a un número creciente de conflictos sociales (Tanaka, 2006; Grompone, 2009). Segundo, la Comisión Verdad y Reconciliación promovió la adopción de una política de indemnización para todas las víctimas del conflicto armado. Entre 1980 y 2000, Perú conoció un conflicto violento interno en el que se enfrentaban, por un lado, el grupo Sendero Luminoso y el Movimiento Túpac Amaru (MRTA) y, por otro, las fuerzas legitimas del Estado (la policía y el ejército). Como consecuencia del conflicto hubo 69.280 víctimas mortales de las cuales el Estado fue responsable del 30\% (CVR 2003).

Las presiones sociales en favor, por un lado, de las nuevas políticas sociales y, por otro, de políticas de indemnización a las víctimas, llevaron al Gobierno a optar por un PTC en el que los primeros beneficiarios fueran las víctimas del conflicto armado, que eran también los más pobres ${ }^{12}$. Como consecuencia, en 2010 un 7,6 \% de la población

\footnotetext{
${ }^{8}$ Creado por el Decreto Supremo no 66 del 3 de abril de 2009.

${ }^{9}$ Entrevista del 13 de agosto 2010 con el Coordinador BJA para el departamento de La Paz.

${ }^{10}$ Decreto Supremo n ${ }^{\circ}$ 032-2005-PCM.

${ }^{11}$ Entre 2001 y 2007, el PIB creció de 6.3\% pero en 2004 la pobreza se quedó al mismo nivel que en 2000, afectando a más del 50\% de la población (CEPAL 2006: 74).

${ }^{12}$ Entrevista del 29 de enero 2010 con un miembro del primer comité directorio de Juntos.
} 
total se benefició del programa Juntos, entre los que estaba el 60\% de la población en extrema pobreza (Cecchini et Madariaga, 2011).

Juntos imita los dos grandes PTCs de la región: Bolsa Familia en Brasil y Oportunidades en Méjico (Francke et Mendoza, 2006) y ha sido evaluado por el Banco Mundial. Juntos transfiere el equivalente de 70 \$US cada dos meses a las madres de niños menores de 19 años si cumplen las condiciones establecidas en materia de salud, nutrición y formalización de identidad (Arroyo, 2010). Las promotoras (la mayoría son mujeres), los maestros y el personal sanitario se encargan de implementar el programa en el campo. Las primeras son las que recolectan los formularios que garantizan el cumplimiento de las condiciones y organizan talleres con las madres receptoras. Los maestros y el personal sanitario vigilan que las condiciones se cumplan en las escuelas y en los centros de salud donde las receptoras deben acudir.

\section{Las representaciones sociales de la pobreza}

\subsection{Bolivia}

En Bolivia, hay dos tipos de discursos que están consensuados entre los actores institucionales de los Bonos. El primero concierne a la revalorización del carácter social de los actores subalternos. El segundo se refiere a una consideración positiva de los bonos como política de redistribución y de erradicación de la pobreza ${ }^{13}$. Como consecuencia, las representaciones sociales construidas por los actores del instrumento PTC reflejan unas relaciones Estado/sociedad de tipo nacional-popular con tintes indigenistas.

La llegada al poder del MAS con el apoyo de los movimientos sociales indígenas, de los campesinos y de la población originaria y de los sindicatos conllevó, en primer lugar, a un discurso en el que se da una revalorización de los actores subalternos. Así, los políticos responsables de las políticas de lucha contra la pobreza dejaron de hablar de los "pobres" y pasaron a hablar de los "indígenas". Por ejemplo, la directora del Plan Vida comentó que un ministro de la planificación del desarrollo había dejado de hablar de pobres y había pasado a referirse a los guerreros indígenas:

El ministro (...) les decía "los pueblos de los ayllus han sido los guerreros, guerreros aimaras, guerreros de los incas, han sido guerreros del olvido de la república y ahora son guerreros".

${ }^{13}$ Para diferenciarse de las políticas neoliberales de "reducción" de la pobreza, el gobierno de Evo Morales habla de "erradicación" de la pobreza, entendida, al menos discursivamente, como la eliminación de las causas estructurales de la pobreza arraigada en las desigualdades sociales y étnicas. 
Ser indígena y no sólo "pobre" provoca una apreciación de la condición social de los actores considerados a los que se ve como los motores del nuevo proyecto político boliviano. Además, si los pobres en tanto que indígenas, pobladores originarios o campesinos, viven en comunidad ${ }^{14}$, ésta se convierte en el lugar donde se debe centrar la atención pública para resolver la pobreza. Por ejemplo, en la cita siguiente de un responsable del PEEP está claro que la pobreza se combate mediante la gestión de tipo comunitario:

El enfoque principal de nuestro plan es un enfoque de gestión comunitaria integral. Es decir, los pobres tienen que manejar la chequera. Eso es uno, y ellos tienen que decidir.

Se trata de tener en cuenta a las comunidades indígenas y de transferirles poder de decisión. Por lo tanto, el papel del Estado se limita a asistir técnica y financieramente a las comunidades para que éstas puedan definir y determinar sus propios proyectos, tal como lo expresa un miembro del PEEP:

Cortar con el paternalismo, ayudar pero no tomar decisiones por los pobres, eso es para nosotros la clave para el éxito para el programa de erradicación de la extrema pobreza (...) la decisión no es nuestra decisión sino la de las comunidades, esta decisión comunal de saber qué hacer con su plata, qué hacer con sus recursos.

Sin embargo, el papel del Estado sigue siendo importante de acuerdo con el principio del "vivir bien", tal como lo expresa un antiguo ministro de la planificación del desarrollo:

Así el vivir bien y hacia lo comunitario, recoge el acto constitucional: fortalecer al Estado y pasar del modelo primario extractivo al modelo productivo (...) [eso] tiene dos cosas fuertes: el enfoque territorial y la articulación entre la economía estatal y las otras formas de organizaciones económicas.

En este argumento, por un lado, la comunidad se eleva al rango de actor decisional principal y, por el otro, se hace al Estado responsable de la transición de un modelo al otro. Además el papel que le corresponde al Estado gira alrededor del "vivir bien". Con este, no se trata más de "vivir mejor", entendido como crecer económicamente y mejorar las condiciones de vida material, sino de "vivir bien" de acuerdo con los preceptos de las comunidades indígenas. Según el mismo actor, en torno al vivir bien se articula todo un modelo económico y ecológico:

Pero también es un modelo económico que se abre a la concepción ecológica (...) sobre los temas de la biodiversidad, recursos naturales definidos estratégica-

${ }^{14}$ El término "comunidad" no ha sido definido nunca claramente y abarca una amplia gama de realidades sociales en las que el denominador común parece ser la organización colectiva y la solidaridad de un grupo de individuos a menudo de origen indígena, nativo o campesino. 
mente para el vivir bien, no mercantilizarlos. (...) El vivir bien es una concepción indígena, aimara, pero también chaqueña (...) El vivir bien construye un modelo cultural y civilizatorio alternativo al capitalismo y a la modernidad.

A partir de estas condiciones, con la finalidad de dar contenido al concepto del "vivir bien", se promueven otros indicadores para medir la pobreza; así, según la directora del "Plan Vida":

Darle un contenido (...) a la propuesta del vivir bien (...) que nosotros no vamos a agarrar los indicadores tradicionales (...). Queremos agarrar desde plan nacional de desarrollo los indicadores de vivir bien, porque justo el contrapuesto es vivir mal, es el clásico que conociéremos como la extrema pobreza.

Esta última cita muestra la debilidad del concepto de "vivir bien" en la medida en que, si su contrario es el "vivir mal" definido como la pobreza extrema, la definición de "vivir bien" es similar al planteamiento "clásico" de salir de la pobreza, del cual quiere justamente distanciarse la noción de "vivir bien". De todo esto resulta que los actores políticos parece que tienen dificultades por construir representaciones que rompan totalmente con aquéllas "clásicas" de la pobreza.

En segundo lugar, otro conjunto discursivo destaca que la lucha contra la pobreza hoy en día pasa por la redistribución de los recursos y el acceso a servicios sociales básicos. Para un investigador de UDAPE ${ }^{15}$ que participó a la elaboración del BJA, la "nacionalización" de los hidrocarburos permite la recuperación de la soberanía nacional y una mayor redistribución económica:

Entonces, lo que se hizo ahora es señalizar (...) que lo que antes iba a otros lugares, porque las empresas eran de otros países, estos recursos, ahora, como son nacionales pueden ser distribuidos entre la gente (...) Ahora todo es propio.

Más precisamente, la redistribución pasa por los bonos, que son vistos como instrumentos eficaces de lucha contra la pobreza, como lo destaca la directora nacional del BJA:

Todo programa de protección social que son los programas bajo los que están inmersos este tipo de bonos, como el bono Juancito Pinto, el bono Juan Azurduy, el bono Dignidad, son medidas que realmente son generadoras de luchas contra la pobreza.

En resumen, en Bolivia, primero hay un proceso de revalorización de los actores subalternos mediante el paso de la figura de "pobre" a la de "indígena". Los pobres, en

${ }^{15}$ Unidad de Análisis de Políticas Sociales y Económicas, que es una institución creada en 1983 dependiente del Ministerio de Planificación del Desarrollo. 
tanto que indígenas, son representados como revolucionarios, como guerreros ${ }^{16} \mathrm{y}$ como garantes del proceso de cambio desde el orden neoliberal hacia la construcción de un modelo social alternativo. Segundo, los Bonos, como políticas resultantes de la "nacionalización" de los hidrocarburos, son considerados por todos los actores como políticas sociales eficaces, porque dan lugar a la redistribución de las ganancias financieras de la "nacionalización" y porque contribuyen a la erradicación de la pobreza.

Se puede entonces destacar que las representaciones consensuales de la pobreza refuerzan el análisis realizado por una serie de autores como Lavaud (2005), Lacroix (2007) y Stefanoni (2009) de que Bolivia está en un proceso de politización de la etnicidad y de etnización de su vida política. El primer proceso concierne a la construcción de organizaciones que se definen según su origen étnico $\left(\mathrm{CIDOB}^{17}, \mathrm{CONAMAK}^{18}\right)$ o campesino y étnico (cocaleros, CSUTCB ${ }^{19}$, Bartolinas Sisas). El segundo proceso empieza en los '90 y se refiere al hecho de que la vida política se polariza por varios etno-regionalismos, por lo que cada agente político "se siente obligado en un momento u otro a posicionarse respecto del clivaje étnico" (Lavaud, 2005: 108). Entonces, la pobreza, como la mayoría de los problemas sociales, se "etnicifica" para politizarse. En otros términos, la pobreza debe pasar por el filtro de la etnicidad para ser reconocida como un problema legítimo en la esfera política.

Sin embargo, parece que las representaciones de los pueblos indígenas y sus comunidades son en gran medida naturalizadas e idealizadas. La imagen del "buen indígena" sustituye a la del "pobre indio ignorante e inútil". Ser indígena es representado con una identidad transparente, unívoca y no problemática. Los discursos parecen construir actores sociales positivos y buenos en sí mismos y no provenientes de relaciones de poder. Estos discursos pertenecen a un cierto esencialismo que idealiza a estos grupos sociales. Varios autores, Stefanoni (2011a), Cassen (2013) y Canessa (2006) hablan de "esencialismo estratégico" del gobierno del MAS en el que el indígena, presentado como un sujeto colectivo homogéneo, es una construcción abstracta positiva. El discur-

${ }^{16}$ Hay que tener en cuenta que no se trata de un actor individual, sino colectivo. Los movimientos sociales (muchos de los cuales afirman su identidad indígena) son los actores centrales del cambio impulsado por el gobierno de Evo Morales.

${ }^{17}$ Confederación de los Pueblos Indígenas de Bolivia, que agrupa a los indígenas de las tierras bajas y que fue creada con el apoyo de Organizaciones No Gubernamentales (ONGs) y de la Iglesia Católica.

${ }^{18}$ Consejo Nacional de Ayllus y Markas del Qullasuyu que agrupa a los originarios de la zona andina. Fue fundada en 1987 con el apoyo de ONGs. Estas últimas sostienen a las organizaciones de carácter étnico en Bolivia porque se inscriben en una tendencia de la cooperación internacional que, desde la caída del muro de Berlín, tiene como objetivo remplazar el clivaje de clases por el étnico, en una lógica de debilitamiento del Estado (Do Alto y Stefanoni, 2008: 65).

${ }^{19}$ Confederación Sindical Única de Trabajadores Campesinos de Bolivia. 
so indigenista e indianista ${ }^{20}$ presenta el autóctono como "dado" al (re)descubrimiento de un proyecto histórico.

Además, el papel asignado al Estado es vago, ambivalente y contradictorio: ¿es el motor del desarrollo o es el apoyo a procesos iniciados desde la sociedad civil por las comunidades? El discurso en favor de la redistribución parece más cercano al planteamiento desarrollista ${ }^{21}$ que pregona la rehabilitación de un Estado fuerte como agente de desarrollo a través de mega proyectos petroquímicos, mineros, carreteros e hidroeléctricos. Este modelo da un papel muy relevante a la explotación de los recursos naturales y a las políticas productivistas que, en realidad, van en contra de la preservación del medio ambiente que está vinculado a la política del "vivir bien". Los acontecimientos del TIPNIS (Territorio Indígena y Parque Isidoro Sécure) muestran que la balanza se acabó inclinando más del lado del "desarrollismo" que del "vivir bien"22.

La politización de la pobreza por su etnicización y el retorno al rol del Estado en lo social conllevan a representarlo como el garante de la solidaridad entre todos los ciudadanos. Sin embargo, se trata de una etnicización esencialista que no permite al Estado reconocer la alteridad indígena. En suma, las relaciones Estado/sociedad basadas en el instrumento de política social que son los bonos remiten a un nacionalismo-popular con tintes indigenistas.

${ }^{20}$ El indigenismo y el indianismo tienen claras diferencias. Favre (1996: 92) define al indigenismo estatal como "la acción sistemática que conduce al Estado por medio de un aparato administrativo especializado para inducir cambios controlados y planificados dentro de la población indígena con el fin de absorber las disparidades culturales, sociales y económicas entre indígenas y no indígenas". Al contrario, el indianismo pretende ser el producto de una reflexión y de una ideología alternativa al indigenismo desde los mismos indígenas. La producción de un discurso indianista es más compleja en la práctica, porque a menudo es resultado de la elaboración de intelectuales o élites indígenas. En Bolivia, el Katarismo es una manifestación explícita del indianismo (Lavaud, 2006). El discurso del MAS en favor de la Pachamama y del vivir bien es a la vez indigenista e indianista. El MAS juega sobre ambos: cuando propone una política para las poblaciones indígenas defiende un discurso indianista, mientras que afirma un discurso indigenista al hacer la representación de las bases indígenas de la sociedad.

${ }^{21}$ El desarrollismo viene de la teoría económica de la CEPAL posterior a la Segunda Guerra Mundial y tiene que ver con la promoción en América Latina del modelo de desarrollo ISI: de industrialización para la sustitución a las importaciones.

${ }^{22}$ En septiembre de 2011, el gobierno hizo uso de la fuerza en contra de movilizaciones populares indígenas en contra de la construcción de una carretera a través del territorio indígena de este parque. Estas posiciones gubernamentales apoyan las declaraciones de julio de 2011 del Vicepresidente que estigmatizaban los portavoces del vivir bien como actores sumisos a ONG ambientalistas occidentales. En otro momento el Vicepresidente declaró sin matices que el derecho del Estado es superior a los derechos territoriales de los pueblos amazónicos con respecto a la explotación de los recursos naturales (Lavaud, 2009: 101). 


\subsection{Perú}

En Perú, los responsables políticos y administrativos de Juntos representan a los pobres como perezosos por naturaleza y responsables de su situación y, en consecuencia, limitan el papel del Estado a desarrollar políticas focalizadas para los "verdaderos" pobres. Por esta razón las relaciones Estado/sociedad se conciben desde una perspectiva individualista y marcadas por el neoliberalismo.

La idea de los pobres como ociosos y responsables de su situación se observa en el próximo extracto de un ginecólogo, antiguamente funcionario del MINSA, a cargo de Juntos en las comunidades dispersas de Ayacucho:

La gente no quiere trabajar porque el jornal promedio era 20 soles y preferían dedicarse a otras cosas que dedicarse a eso. Entonces que digan que no hay trabajo, a mí me parece que no es así (...) se han acostumbrado a trabajar sólo para su supervivencia, o sea, son personas demasiado conformistas, tienen para alimentarse, y ya está.

Según esta cita, el problema de la pobreza no se sitúa en la falta de empleos sino en el hecho de que los pobres no quieren trabajar. Según este médico, los pobres deberían someterse a las condiciones que marca el mercado y cambiar de mentalidad para demostrar su voluntad de mejorar su situación socioeconómica. En el mismo orden de ideas, los programas sociales son vistos como reproductores de esta ociosidad de los pobres, tal como lo expresa un profesor encargado de Juntos de un pequeño centro urbano de Ayacucho ${ }^{23}$ :

El campesino es más perezoso, trabaja menos (...) desde [la existencia del] programa Juntos.

Sin embargo la focalización también es vista como el mejor método para ayudar a los pobres que quieren salir adelante (Vasquez, 2006; Monge et al., 2009). La focalización permite "obtener el mejor impacto en términos de reducción de la pobreza por cada nuevo sol24 invertido" (Monge et al., 2009: 12). En el momento en el que tiene que realizarse una selección de pobres, es cuando se construyen y distinguen las categorías de los "verdaderos" pobres, que pueden beneficiarse de los programas, y los otros - los infiltrados. Estos últimos son vistos como "aprovechados": personas sin necesidades que están en el programa. Así lo explica un funcionario responsable del programa Juntos en una pequeña comunidad de Ayacucho:

${ }^{23}$ Para preservar el anonimato de los entrevistados no se revela el nombre de las comunidades o centros urbanos en donde se realizó el trabajo de campo.

${ }^{24}$ El nuevo sol es la moneda peruana. 
Hay muchas personas que a pesar de que tienen algo en la casa [para] darle de comer a sus hijos, están beneficiándose. (...) gente que tiene. Adentro [hay] gente que tiene (...) No deberían estar.

Ahora bien el proceso de filtración y selección tiene el corolario de la sub-cobertura que resulta del programa, es decir, la existencia de "verdaderos" pobres que quedan excluidos del programa, como lo expresa otra responsable de la misma pequeña comunidad de Ayacucho:

Hay otros más necesitados (...) más necesitados pero no han entrado...

Esta representación consensuada de la focalización como principio director de las políticas sociales remite a una representación más amplia de los mecanismos de regulación social que concede un peso preponderante al mercado. Según esta las políticas sociales solo deben dirigirse a las poblaciones en extrema necesidad porque los primeros mecanismos de distribución de la riqueza son el mercado y el crecimiento económico. Este planteamiento se explicita en las próximas observaciones de un alto funcionario del Ministerio de Desarrollo Social a cargo de Juntos:

La concepción normal (...) una concepción típica (...) el mercado distribuye, el mercado asigna los recursos, y asignando esos recursos ... y además es una idea muy difundida de que ese sistema capitalista incluye, es muy inclusivo.

La regulación de la sociedad por el mercado está representada como lo "normal", es decir, la única posible. Una focalización específica eficiente que esté asociada al objetivo del crecimiento económico se percibe como la clave de la lucha contra la pobreza. El papel del Estado se limita, entonces, a ofrecer una determinada igualdad mínima inicial, no de resultados. El Estado no pretende resolver las desigualdades sociales a través de los programas sociales, estas constituyen problemas percibidos como insuperables, tal como lo explica el mismo actor:

Pero la desigualdad es un tema más difícil porque las propias brechas al interior de los tramos inferiores de desigualdad son grandes, incluso más grandes que las que están arriba (...) [es entonces] en este 20\% que están muy cerca de salir de la línea de pobreza, alli los programas tiene mayor éxito.

En otras palabras, este alto responsable explica que no es "rentable" invertir en las poblaciones más pobres. Según él, la responsabilidad social del Estado se concreta en apoyar a la parte de la población que tiene el potencial de salir de la pobreza. Concentrar los programas sociales sobre los menos pobres de entre los pobres es lo que permite, paradójicamente, una mayor reducción de la pobreza. Este razonamiento desliga completamente las cuestiones de la pobreza y de la desigualdad. Mientras que las desigualdades entre los pobres permanecen como son, será cada vez más ventajoso focalizarse en los menos pobres del conjunto de los pobres (Lautier, 2002: 148-150). 
La focalización así entendida permite tratar a la pobreza como un problema técnico, sacándola del ámbito de las relaciones de poder y de las negociaciones entre los actores sociales y políticos, tales como los sindicatos, los partidos políticos y los receptores de las políticas sociales. Entonces una de sus principales consecuencias concierne a la individualización que las políticas sociales producen, pues la responsabilidad individual (del perceptor de la ayuda) es elevada al grado de valor dogmático en detrimento del valor de la solidaridad. En estas condiciones, más que representar el interés general y la cohesión de la comunidad política a nivel nacional, el Estado aparece como un agente que potencialmente genera divisiones. Dicho de otro modo, más que solucionar el problema público de la "pobreza", las políticas de lucha contra la pobreza crean una nueva categoría social: los pobres.

Además, la focalización tiende a despolitizar la pobreza. Ningún debate en la esfera política discute los criterios de selección de la población elegida. Parece evidente que los grupos más vulnerables, que son los que se encuentran en extrema pobreza, como los niños, las mujeres víctimas de violencia, las personas mayores y los trabajadores pobres quedan ausentes (Vargas, 2010: 16). Como resultado de todo ello, los criterios de cálculo de la pobreza y la focalización llevan a una manera de concebir el problema de la pobreza en la que se eliminan los debates estructurales sobre, por ejemplo, la redistribución de la riqueza o el empleo (Mauro, 2011: 243). También se excluye el debate sobre las relaciones sociales de producción en las que las poblaciones están insertas y que son las que generan la pobreza (Bey, 2003: 21).

Estas representaciones de la pobreza remiten a una consideración de las relaciones Estado/sociedad propias del enfoque de la "inversión social" de raíz neoliberal. De hecho, desde hace una veintena de años las instituciones públicas de Perú han estado invadidas, especialmente en el ámbito económico, por lo que Gonzales (2007: 30) denomina "la corporación de los economistas neoclásicos" 25 . Esta "casta científica" se ha formado principalmente en las grandes universidades estadunidenses y ha hecho carrera en la función pública, en las organizaciones internacionales (el FMI, BM, BID) $\mathrm{y}$ en los institutos de investigación especializados. Esta reivindica el monopolio del discurso legítimo en este ámbito, realizando un peritaje de la pobreza en el que el instrumento clave de objetivación es la herramienta estadística (Adrianzen, 2010: 43; Vergara, 2012).

${ }^{25}$ Por ejemplo, los cuatro Ministros (Luis Carranza, Luis Valdivieso, Mercedes Aráoz e Ismael Benavides) que se sucedieron en el Ministerio de Economía y Finanzas bajo el gobierno de Alan García (2006-2011) son graduados de universidades estadunidense ortodoxas. El segundo estuvo encargado de una carrera en el Fondo Monetario Internacional (FMI) durante 28 años. De los principio defendidos por esta institución extrajo las premisas para su política económica durante la crisis económica de 2008 que valió al Perú la mayor reducción del índice de crecimiento de la región —pasó del 9,8\% del PIB en 2008 al 0,9\% del PIB en 2009- (Cruzado y Sotelo 2011: 258). 
Una razón de la fortaleza del neoliberalismo en Perú se encuentra en su implementación por un régimen autoritario: el fujimorismo. Después de su autogolpe de $1992 \mathrm{Fu}-$ jimori implementó unas políticas de ajuste estructural de las más agresivas de la región; entre otras razones porque las fuerzas de resistencia social estaban muy debilitadas por la guerra interna. El grupo Sendero Luminoso derrocó a los líderes populares — sindicalistas, indígenas y campesinos - que negaban su poder y los supervivientes fueron para el gobierno de Fujimori sospechosos de ser terroristas y, como consecuencia, potencialmente ejecutables. La debilidad de la politización del clivaje étnico y campesino en Perú impidió el desarrollo de movimientos sociales amplios, como en Bolivia o Ecuador, que desafiaran al neoliberalismo. Además, para el gobierno de Fujimori todo oponente al neoliberalismo era considerado como terrorista, legitimando así una creciente criminalización de los movimientos sociales y su represión por el Estado ${ }^{26}$.

Aunque la vuelta a la democracia se ha acompañado de una mayor transparencia institucional, sin embargo los fundamentos del neoliberalismo fujimorista han seguido siendo poderosos. El Presidente Alan García reemplazó en los círculos de poder económico, político y comunicacional del $\mathrm{APRA}^{27}$ a los intelectuales universitarios, cuya vinculación viene desde los años veinte, por tecnócratas "neoclásicos". En expresión de Cotler (2008: 389) en el APRA se ha producido de esta forma una reconversión casi religiosa al neoliberalismo contradiciendo sus líneas ideológicas históricas. Así lo atestiguan las manifestaciones del Presidente contra el "perro del hortelano". Los grupos en contra de la explotación de los recursos naturales por grandes empresas privadas, sobre todo extranjeras, son calificados por el Presidente en varios artículos ${ }^{28}$ como "perros del hortelano" y presentados como enemigos de la democracia y de la modernidad. Además, detrás de este discurso neoliberal amargo se esconde un racismo apenas disimulado (Cotler, 2008: 393).

${ }^{26}$ Como en los acontecimientos de Bagua en junio de 2009. En esta provincia las comunidades amazónicas protestaban desde mediados de 2008 contra una serie de decretos que regulan la posesión y la concesión de tierras y, en particular, los derechos de propiedad de tierra de comunidades indígenas en el marco de la implementación del Tratado de libre comercio con los Estados Unidos. El gobierno rechazaba todo diálogo lo que condujo a los acontecimientos, conocidos como el "Baguazo", de confrontación entre la policía y grupos indígenas que causó 34 muertos (García, 2011: 199).

${ }^{27}$ Alianza Popular Revolucionaria Americana.

${ }^{28}$ Estos artículos fueron publicado por El Comercio entre octubre 2007 y marzo 2008: "El síndrome del perro del hortelano" del 28.10.2007; "Receta para acabar con el perro del hortelano" del 25.11.2007 y "El perro del hortelano contra el pobre" del 02.03.2008. 


\section{Conclusiones}

Para concluir, se subrayan las diferencias encontradas entre Perú y Bolivia a partir del análisis de la aplicación de un instrumento similar de política de lucha contra la pobreza: los programas de transferencias condicionadas ${ }^{29}$. El análisis de discurso permite demostrar las diferencias de las representaciones de la pobreza y del papel del Estado entre los actores bolivianos y peruanos.

Si bien las representaciones de la pobreza son homogéneas en los dos países, están contrapuestas entre ambos. Tales distinciones y oposiciones deben ponerse en contexto tomando como referencia las diferencias socio-políticas e históricas: mientras Perú permanece como uno de los países más neoliberales de Sudamérica, Bolivia trata de romper con este régimen político y económico.

Como se ve en el análisis realizado, Bolivia ha enfatizado un proceso de etnicización de su política, que coloca la imagen del indígena al frente de la escena pública y política. No solamente las cuestiones indígenas se politizan, sino que también la vida política se "etnitiza". En otras palabras, la politización de un problema social pasa por el filtro del clivaje étnico. La pobreza no escapa al fenómeno. Además, al acercarse a las ideas del nacionalismo popular, los discursos bolivianos se acompañan de una representación de la pobreza estructural cuya solución se encuentra en la redistribución. El consenso se articula, entonces, en torno a una imagen indígena de la pobreza y sobre medidas de redistribución como los bonos. Por lo tanto, el indigenismo de Evo Morales se utiliza como recurso estratégico para oponerse al capitalismo global y para homogenizar el pueblo (Canessa, 2006: 252; Cassen, 2013). Sin embargo, el problema de esta indigeneidad es el que borra, de nuevo, las diferencias. De hecho, la representación del indígena es esencialista. Es decir, "si todo el mundo es indígena, se deja poco lugar para que grupos culturalmente distintos puedan organizar su indigenidad. Por ello una de las paradojas del éxito de las políticas a favor de los indígenas en Bolivia puede llegar a ser la continuidad de la marginación del pueblo indígena" (Canessa, 2006: 259).

La situación peruana se opone en gran medida a la boliviana. Debido a numerosos factores socio-históricos y políticos la politización del clivaje étnico en Perú es escasa y la difusión del neoliberalismo es amplia y poco cuestionada. En el Perú la ola de protestas sociales contra el neoliberalismo, asociando características y símbolos campesinoindígenas a la defensa del medio ambiente, no es percibida (aún) como un amplio movimiento social portador de proyectos políticos alternativos. Paradójicamente, mientras que el Perú alberga una de las más viejas tradiciones políticas de izquierda en la región (el APRA) y una de las mayores poblaciones autóctonas de los países andinos, no dispone ni de un gobierno progresista, ni tampoco de amplios movimientos indígenas a nivel nacional, lo que lo diferencia claramente frente a Bolivia y Ecuador (Pajuelo,

\footnotetext{
${ }^{29}$ Verse el anexo 1 para un esquema que resume estas diferencias.
} 
2010: 319-320). Es por esto por lo que las representaciones consensuales de la pobreza giran alrededor de la responsabilidad individual y de la focalización.

Por lo tanto, si en Bolivia las representaciones de la pobreza parecen tender hacia relaciones Estado/sociedad de tipo nacional-popular basado en un indigenismo estratégico, en Perú se mantienen relaciones más individualistas marcadas por el neoliberalismo.

\section{Bibliografía}

Adrianzén, A. (2010): “Sistemas, minorías y centralización política”, en VV.AA, Perú Hoy. Centralismo y concentración, Lima, DESCO, pp. 33-48.

ARroyo, J. (2010): Estudio cualitativo de los efectos del programa JUNTOS en los cambios de comportamiento de los hogares beneficiarios en el distrito de Chuschi: avances y evidencias, Lima, Programa Juntos.

Barrientos, A. y J. DeJong (2006): "Reducing Child Poverty with Cash Transfers: A Sure Thing?", Development Policy Review, 24 (5), pp. 537-552.

Barrientos, A. (2009): "Labour markets and the (hyphenated) welfare regime in Latin America", Economy and Society, 38 (1), pp. 81-108.

BEy, M. (2003): "Pérou: quelles politiques de lutte contre la pauvreté?", Autrepart. IRD, 27, pp. 5-23.

Bourdieu, P. (1982): Ce que parler veut dire, Paris, Fayard.

Canvire-Bacarreza, G. y M. Mariscal Ayaviri (2010): Políticas macroeconómicas, choques externos y protección social en Bolivia, La Paz, UDAPE.

CANEssa, A. (2006): "Todos somos indígenas: Towards a New Language of National Political Identity", Bulletin of Latin American Research, 25 (2), pp. 241-263.

CAssen, C. (2013): "La figura del indígena como encarnación del pueblo boliviano: discusión en torno al esencialismo estratégico del Movimiento al Socialismo (MAS)", Rubrica Contemporanea, 2 (3), pp. 67-82.

Cecchini, S. y A. Madariaga (2011): Programas de transferencias condicionadas. Balance de la experiencia reciente en América latina y el Caribe, Santiago de Chile, CEPAL, ASDI.

CEPAL (2006): Estadisticas sociales, Santiago de Chile, CEPAL.

Cotler, J. (2008): “Capitalismo y democracia en el Perú: la tentación autoritaria”, en A. Rodrich, J. De Belaunde y J. Cotler, Perú en el siglo XXI, Lima, PUCP, pp. 361-394.

Cruzado, E. y V. Sotelo (2011): "Políticas fiscales y pereza gubernamental”, en VV.AA., Perú Hoy. El quinquenio perdido. Crecimiento con exclusión, Lima, DESCO, pp. 252-263.

CVR (2003): Informe final, Lima, Comisión de Verdad y Reconciliación.

Do Alto, H. y P. Stefanoni (2008): Nous serons des millions, Paris, Raison d'agir. 
FAVRe, H. (1996): L'indigénisme, Paris, Presses Universitaires de France.

Fiszbein, A. y N. Schady (eds.) (2009): Conditional Cash Transfers. Reducing Present and Future Poverty, Washington, D.C., World Bank.

Francke, P. y A. Mendoza (2006): "Perú: programa JUNTOS”, en Ernesto Cohen y Rolando Franco (eds.), Transferencias con coresponsabilidad. Una mirada latinoamericana, Lima, FLACSO, pp. 389-432.

GARCÍA, M. (2011):"Gobernar al decretazo: los peruanos del hortelano y los límites del modelo de Alan García" en VV.AA, Perú Hoy. El quinquenio perdido. Crecimiento con exclusión. Lima, DESCO, pp. 184-204.

GonZales de Olarte, E. (2007): "La economía política peruana de la era neoliberal 19902006”, en Murakami, Yusuke (ed.), Dinámica politico-economica de los países andinos, Universidad de Kyoto, CIAS/IEP, pp. 295-342.

Gray Molina, G. (2007): "El reto posneoliberal de Bolivia”, Nueva Sociedad, 209, pp. 118-129.

Grompone, R. (2009): Entre el crecimiento económico y la insatisfacción social: las protestas sociales en el Perú, Lima, IEP.

Henning, E., W. van RensBurg y B. SMit (2004): Finding your way in Qualitative Research, Pretoria, Van Schaik.

Jenson, J. (2010): "Diffusing Ideas for After Neoliberalism: The Social Investment Perspective in Europe and Latin America", Global Social Policy, 10 (1), pp. 59-84.

Jenson, J. y D. SAint-Martin (2006): "Building blocks for a new social architecture: the LEGOTM paradigm of an active society", Policy \& Politics, 34 (3), pp. 429-451.

Jodelet, D. (1989): Les représentations sociales, Paris, P.U.F.

Lacroix, L. (2007): “La gouvernance de l'ethnicité en Bolivie”, Outre-Terre, 1, pp. 253-271.

Lascoumes, P. y P. Le Galés (2005): Gouverner par les instruments, Paris, Presse de Science Po.

Lautier, B. (2002): "Pourquoi faut-il aider les pauvres? Une étude critique du discours de la Banque Mondiale sur la pauvreté", Tiers-Monde, 43 (169), pp. 137-165.

Lavaud, J. P. (2005): "Démocratie et ethnicisation en Bolivie", Problèmes d'Amérique latine, 56, pp. 105-128.

Lavaud, J. P. (2006): "Bolivie: vers l'anarchie segmentaire? L' "ethnicisation" de la vie politique", Hérodote, 4 (123), pp. 62-81.

LAVAUD, J. P. (2009): "Indianisme et écologie dans les pays andins: dispositif légal, discours officiels et mobilisations", Problèmes d'Amérique Latine, 76, pp. 97-117.

Marco Navarro, F. (2013): El Bono Juancito Pinto del Estado Plurinacional de Bolivia. Programas de transferencias monetarias e infancia, Santiago de Chile, CEPAL.

Martinez Franzoni, J. (2008): "Welfare regimes in Latin America: capturing constellations of markets, families and policies", Latin American Politics and Society, 50 (2), pp. 67-100. 
Mauro, R. (2011): “Empleo, pobreza y democracia: el eslabón escondido", en VV.AA., Perú hoy. El quinquenio perdido. Crecimiento con exclusión. Lima, DESCO, pp. 229-249.

Monge, A., E. VÁsquez y D. WinkelRied (2009): ¿Es el gasto social público en programas sociales regresivo en el Perú?, Lima, CIES, Universidad del Pacífico.

Morel, N., B. Palier y J. Palme (2011): Towards a Social Investment Welfare State? Ideas, Policies and Challenges, Bristol, Policy Press.

Nagels, N. (2013): Genre et politiques de lutte contre la pauvreté au Pérou et en Bolivie: quels enjeux de citoyenneté?, Tesis doctoral inédita, Graduate Institute, Ginebra.

Nagels, N. (2014a): "Programmes de transferts conditionnés au Pérou et en Bolivie: entre ciblage et universalisation de l'assistance sociale", Revue internationale de politique comparée, 21, pp. 111-132.

Nagels, N. (2014b): "Social Investment Perspective, Gender and Conditional Cash Transfer Programs in Peru and Bolivia", en Rocha Lukic, M. y C., Tomazini (eds.), Analyzing Public Policies in Latin America: A Cognitive Approach, Newcastle upon Tyne, Cambridge Scholars Publishing, pp. 1-24.

Nagels, N. (2014c): "Programmes de transferts conditionnés et santé maternelle au Pérou et en Bolivie", Les cahiers de santé publique et de protection sociale, 15, pp. 61-69.

Nagels, N. (en prensa), "The Social Investment Perspective, Conditional Cash Transfer Programs and the Welfare Mix: Peru and Bolivia", Social Policy and Poltics.

OIT (2011): Social Protection Floor for Fair and Inclusive Globalization. Report of the Advisory Group chaired by Michelle Bachelet Convened by the ILO with the collaboration of the WHO, Geneva, Organisation Internationale du Travail (Bachelet Report).

Pajuelo, R. (2010): “Movimientos indígenas y política nacional en los Andes: ideas para un balance", en VV.AA., Perú hoy. Desarrollo, democracia y otras fantasías. Lima, DESCO, pp. 298-320.

Quivy, R. y L. VAN CAMPENHOUdT (1995): Manuel de recherche en sciences sociales, Paris, DUNOD.

RaZAVI, S. (2014):"Addressing/reforming care, but on whose terms?", en Shirin M. Ray and Georgina Waylen (eds.), New Frontiers in Feminist Political Economy, Milton Park, Routledge, pp. 114-134.

Skoufias, E. y B. McClafferty (2001): Is PROGRESA Working? Summary of the Results of an Evaluation, Washington D.C, International Food Policy Research Institute (IFPRI).

StaAB, S. (2010): "Social Investment Perspective in Chile and Latin America: Towards Equal Opportunities for Women and Children?”, Journal of Social Policy, 39 (4), pp. 607-626.

Stefanoni, P. (2009): “"Indianisation” du nationalisme ou refondation permanente de la Bolivie", Alternatives Sud, 16 (3), pp. 27-44. 
Stefanoni, P. (2011): La Bolivie d'Evo Morales entre progrès et désenchantement, Louvainla-Neuve, CETRI.

SugiYama, N. (2011): "The diffusion of Conditional Cash Transfer programs in the Americas", Global Social Policy, 11 (2-3), pp. 250-278.

TanaKa, M. (2006): "Las protestas sociales en Perú, entre fujimorismo y la "transición democrática (1995-2004)”, en Massal (ed.), ¿Representaciones o participación? Los retos y desencantos andinos en el siglo XXI, Bogotá, IEPRI/IFEA, pp. 113-139.

THE ECONOMist (2010): "How to get children out of jobs and into school. The limits of Brazil's much admired and emulated anti-poverty programme", The Economist, 29 de julio.

Unidad Ejecutiva del Bono Juancito Pinto (2008): Informe de cierre preliminar del Bono Juancito Pinto al 31 de diciembre 2008, La Paz, Ministerio de Educación y de Cultura.

Vargas, R. (2010): Gender risk, poverty and vulnerability in Peru. A case study of the Juntos programme, London, ODI.

VÁsquez Huamán, E. (2006): Programas sociales: ¿de lucha contra la pobreza?: casos emblemáticos, Lima, CIES, Universidad del Pacífico.

Villatoro P. (2004): Programas de reducción de la pobreza en América latina. Un análisis de cinco experiencias, Santiago de Chile, CEPAL.

Weisbrot, M., R. Ray y J. Johnston (2009): Bolivia: The Economy During the Morales Administration, Washington D.C, Center for Economic and Policy Research.

\section{Anexos}

TABLA 1:ESQUEMA DE LAS DIFERENCIAS ENTRE LAS REPRESENTACIONES SOCIALES DE LOS PTC BOLIVIANOS Y PERUANOS

\begin{tabular}{lll}
\hline & Bolivia & Perú \\
\hline Pobres & Indígenas idealizado, valiosos & Ociosos \\
Estado & Fuerte, « desarrollista », distributor & Focalizador, mercantilista \\
Pobreza & Politizada & Individualizada \\
Relaciones Estado / sociedad & Nacional-populares & Neoliberales \\
\hline
\end{tabular}

Fuente: Elaboración propia. 